\title{
On some types of slight homogeneity
}

\author{
Samer Al Ghour, Nahed Al Khatib \\ Department of Mathematics and Statistics, Jordan University of Science and Technology, Jordan
}

\begin{tabular}{l} 
Article Info \\
\hline Article history: \\
Received Sep 6, 2019 \\
Revised Feb 21, 2020 \\
Accepted Feb 29, 2020 \\
\hline
\end{tabular}

Keywords:

Clopen sets

Slight continuous

Slight homogeneity

\begin{abstract}
As a generalization of the concept SLH space, we introduce the concept of slightly strongly locally homogeneous (SSLH) spaces. Also, we introduce the concepts of slightly dense set as well as slightly separable space, and use them to introduce two new types of slightly countable dense homogeneous spaces. Several results, relationships, examples and counter-examples concerning these concepts are obtained.
\end{abstract}

Corresponding Author:

Samer Al Ghour,

Department of Mathematics and Statistics,

Jordan University of Science and Technology,

Irbid 22110, Jordan.

Email: algore@just.edu.jo

\section{INTRODUCTION}

Throughout this paper by a space we mean a topological space. Let $(X, \tau)$ be a space. $(X, \tau)$ is homogeneous [1] if for any two points $x, y \in X$ there exists a homeomorphism $f:(X, \tau) \rightarrow(X, \tau)$ such that $f(x)=y \cdot(X, \tau)$ is strongly locally homogeneous (abbreviated: SLH) [2] if $\tau$ has a base $\sigma$ such that for each $A \in \sigma$ and points $x, y \in A$, there exists a homeomorphism $f:(X, \tau) \rightarrow(X, \tau)$ such that $f(x)=y$ and $\left.f\right|_{X-A}$ equal to the identity. A separable space $(X, \tau)$ is countable dense homogeneous (abbreviated: $\mathrm{CDH})$ [3] if given any two countable dense subsets $A, B$ of $X$, there is a homeomorphism $f:(X, \tau) \rightarrow(X, \tau)$ such that $f(A)=B$. [4-17] are some recent works related to the above concepts. Let $f:\left(X, \tau_{1}\right) \rightarrow\left(Y, \tau_{2}\right)$ be a function. Then $f$ is slightly continuous [18] if the inverse image of every clopen subset of $\left(Y, \tau_{2}\right)$ is a clopen subset of $\left(X, \tau_{1}\right) . f$ is slightly homeomorphism [19] if $f$ is a bijection and $f$ and $f^{-1}$ are slightly continuous. There are some papers in the literature where the definition of a homogeneous space is modified in the manner that the role of homeomorphisms is given to prehomeomorphisms ([20] and [21]) or semihomeomorphisms [22]. In [19], the authors used slightly homeomorphisms to introduce slightly homogeneous spaces and slightly countable dense homogeneous spaces. In this paper, we will use slightly homeomorphisms to introduce slightly strongly homgeneous spaces and two new types of slightly countable dense homogeneous spaces. Throughout this paper, for any space $(X, \tau)$ and $A \subseteq X,|A|$ will denote the cardinality of $X$ and $\tau_{A}$ will denote the subspace topology on $A$ relative to $\tau$. Moreover, for a subset $A$ of $\mathbb{R}$ we write $\left(A, \tau_{u}\right)$ to denote the subspace topology on A relative to the usual topology. For a non-empty set $X, \tau_{\text {disc }}$ will denote the discrete on $X$.

PROPOSITION 1.1. [19] If $f:\left(X, \tau_{1}\right) \rightarrow\left(Y, \tau_{2}\right)$ is a bijective function such that $\left(X, \tau_{1}\right)$ and $\left(Y, \tau_{2}\right)$ are both connected, then $f$ is a slight homeomorphism.

PROPOSITION 1.2. [19] Every homeomorphism is slight homeomorphism.

PROPOSITION 1.3. [23] Every connected SLH space is homogeneous. 
PROPOSITION 1.4. [19] If $f:\left(X, \tau_{1}\right) \rightarrow\left(Y, \tau_{2}\right)$ is a slight homeomorphism such that $\left(X, \tau_{1}\right)$ and $\left(Y, \tau_{2}\right)$ are zero dimensional spaces, then $f$ is a homeomorphism.

Definition 1.5. [19] A space $(X, \tau)$ is said to be slightly homogeneous if for any two points $x, y \in X$, there exists a slight homeomorphism $f:(X, \tau) \rightarrow(X, \tau)$ such that $f(x)=y$. A subset of a space $(X, \tau)$, which has the form , $S C_{x}=\{y \in X$ : there is a slight homeomorphism $f:(X, \tau) \rightarrow(X, \tau)$ such that $f(x)=y\}$ is called the slightly homogeneous component of $X$ at $x$.

Definition 1.6. [19] A separable space $(X, \tau)$ is said to be slightly countable dense homogeneous (abbreviated: $\mathrm{SCDH}$ ) if given any two countable dense subsets $A, B$ of $X$, there is a slight homeomorphism $f:(X, \tau) \rightarrow(X, \tau)$ such that $f(A)=B$.

PROPOSITION 1.7. [24] Every zero-dimensional CDH space is SLH.

PROPOSITION 1.8. (a) [19] Every CDH space is SCDH but not conversely. (b) [4] Every zero-dimensional SCDH space is CDH.

PROPOSITION 1.9. [19] Every zero-dimensional slightly homogeneous space is homogeneous

PROPOSITION 1.10. [24] Every zero dimensional homogeneous space that is $T_{0}$ is SLH.

PROPOSITION 1.11. [19] If $f:\left(X, \tau_{1}\right) \rightarrow\left(Y, \tau_{2}\right)$ is a slight homeomorphism, $A$ is a clopen subset of $X$ and $f(A)$ is a clopen subset of $\mathrm{Y}$, then the restriction function on $A,\left.f\right|_{A}:\left(A,\left(\tau_{1}\right)_{A}\right) \rightarrow\left(f(A),\left(\tau_{2}\right)_{f(A)}\right)$ is a slight homeomorphism.

PROPOSITION 1.12. [19] Let $(X, \tau)$ be a space and $A$ be a clopen subset of $X$. If $f_{1}:\left(A, \tau_{A}\right) \rightarrow\left(A, \tau_{A}\right)$ and $f_{2}:\left(X-A, \tau_{X-A}\right) \rightarrow\left(X-A, \tau_{X-A}\right)$ are slight homeomorphisms. Then $f_{1} \cup f_{2}:(X, \tau) \rightarrow(X, \tau)$ is a slight homeomorphism.

PROPOSITION 1.13. [19] Let $U$ be a non-empty clopen subset of a space $(X, \tau)$. If $S C_{x}$ is a slightly homogeneous component of $\mathrm{x} \in \mathrm{X}$ and $U \subseteq S C_{x}$, then $S C_{x}$ is open in $X$.

PROPOSITION 1.14. [23] $\left(\mathbb{R}, \tau_{u}\right)$ is a $\mathrm{CDH}$ space.

PROPOSITION 1.15. [23] $\left(\mathbb{Q}^{c}, \tau_{u}\right)$ is $\mathrm{CDH}$.

Definition 1.16. [25] Let $\left\{\left(X_{\alpha}, \tau_{\alpha}\right): \alpha \in \Lambda\right\}$ be a collection of spaces such that $X_{\alpha} \cap X_{\beta}=\emptyset$ for all $\alpha \neq \beta$. Let $X=U X_{\alpha}$ be topologized by $\left\{U \subseteq X: U \cap X_{\alpha} \in \tau_{\alpha}\right.$ for all $\left.\alpha \in \Lambda\right\}$. Then $(X, \tau)$ is called the disjoint sum of the spaces $\left(X_{\alpha}, \tau_{\alpha}\right), \alpha \in \Lambda$.

PROPOSITION 1.17. [19] Let $\left\{\left(X_{\alpha}, \tau_{\alpha}\right): \alpha \in \Lambda\right\}$ be a collection of slightly homogeneous spaces with $X_{\alpha} \cap$ $X_{\beta}=\varnothing$ and $\left(X_{\alpha}, \tau_{\alpha}\right)$ is slightly homeomorphic to $\left(X_{\beta}, \tau_{\beta}\right)$ for all $\alpha, \beta \in \Lambda$. Then the disjoint sum of the spaces $\left\{\left(X_{\alpha}, \tau_{\alpha}\right): \alpha \in \Lambda\right\}$ is slightly homogenous.

PROPOSITION 1.18. [19] Every connected space is slightly homogeneous.

\section{SLIGHTLY STRONGLY LOCALLY HOMOGENEOUS SPACES}

Definition 2.1. A space $(X, \tau)$ is said to be slightly strongly locally homogeneous (SSLH) if for all $p \in X$ and $U$ an open neighborhood of $p$, then there is an open set $V$ in $X$ with $p \in V \subseteq U$ such that if $q \in V$, there exists a slight homeomorphism $h:(X, \tau) \rightarrow(X, \tau)$ such that $h(p)=q$ and $h(x)=x$ for all $x \in X-V$.

THEOREM 2.2. Being "SSLH" is a topological property.

Proof. Let $\left(X, \tau_{1}\right)$ be an SSLH space and let $\left(Y, \tau_{2}\right)$ be any space homeomorphic to $\left(X, \tau_{1}\right)$. Let $y_{0} \in Y$ and let $U$ be an open subset of $Y$ with $y_{0} \in U$. Let $f:\left(X, \tau_{1}\right) \rightarrow\left(Y, \tau_{2}\right)$ be a homeomorphism. Then $f^{-1}\left(y_{0}\right) \in$ $f^{-1}(U)$, which is open in $X$. Since $\left(X, \tau_{1}\right)$ is SSLH, there is an open set $W$ of $X$ with $f^{-1}\left(y_{0}\right) \in W \subseteq f^{-1}(U)$ such that if $q \in W$, then there is a slight homeomorphism $h:\left(X, \tau_{1}\right) \rightarrow\left(X, \tau_{1}\right)$ such that $h\left(f^{-1}\left(y_{0}\right)\right)=q$ and $h(x)=x$ for all $x \in X-W$. Take $V=f(W)$. Then $V$ is open in $Y$ with $y_{0} \in V$. Let $y_{1} \in V$. Then $f^{-1}\left(y_{1}\right) \in$ $W$ and so there is a slight homeomorphism $h:\left(X, \tau_{1}\right) \rightarrow\left(X, \tau_{1}\right)$ such that $h\left(f^{-1}\left(y_{0}\right)\right)=f^{-1}\left(y_{1}\right)$ and $h(x)=$ $x$ for all $x \in X-W$. Define $g:\left(Y, \tau_{2}\right) \rightarrow\left(Y, \tau_{2}\right)$ by $g(y)=\left(f \circ h \circ f^{-1}\right)(y)$. Then $g$ is a slight homeomorphism such that $g\left(y_{0}\right)=y_{1}$ and if $y \in Y-V=f(X-W)$, then $h\left(f^{-1}(y)\right)=f^{-1}(y)$, which implies $g(y)=y$.

THEOREM 2.3. Every connected space is SSLH.

Proof. Let $(X, \tau)$ be a connected space. Let $x \in X$ and $U$ be an open neighborhood of $x$. Take $V=U$. Let $y \in$ $V$. Define $f:(X, \tau) \rightarrow(X, \tau)$ by $f(x)=y, f(y)=x$ and $f(t)=t$ for $t \in X-\{x, y\}$. Since $(X, \tau)$ is connected, by Proposition 1.1, $f$ is a slight homeomorphism. Also, $f(x)=y$ and $f(t)=t$ for all $t \in X-V$. Hence $(X, \tau)$ is SSLH.

The following example shows that the converse of Theorem 2.3 is not true in general.

Example 2.4. Consider the set $X=\{a, b, c\}$ with the topology $\tau=\{\varnothing, X,\{a\},\{b, c\}\}$. Then $(X, \tau)$ is a disconnected SSLH space.

Example 2.4, also is an example of SSLH space that is not slightly homogeneous. 
THEOREM 2.5. Every SLH space is SSLH.

Proof. Let $(X, \tau)$ be an SLH space. Let $x \in X$ and let $U$ be an open neighborhood of $x$. Then there exists an open neighborhood $V$ of $x$ such that $x \in V \subseteq U$ and for every $y \in V$, there is a homeomorphism $f:(X, \tau) \rightarrow$ $(X, \tau)$ such that $f(x)=y$ and $f(t)=t$ for all $t \in X-V$. By Proposition 1.2, $\mathrm{f}$ is a slight homeomorphism. Therefore, $(X, \tau)$ is SSLH

The space $\left([0,1], \tau_{u}\right)$ is connected and by Theorem 2.3, it is SSLH. On the other hand, by Proposition 1.3 it is not SLH. So, the converse of Theorem 2.5 is not true in general. However, we have the following result.

THEOREM 2.6. Let $(X, \tau)$ be a zero dimensional space. If $(X, \tau)$ is SSLH, then it is SLH.

Proof. Let $(X, \tau)$ be a zero dimensional SSLH space. Let $x \in X$ and $U$ an open neighborhood of $x$. Then there exists an open neighborhood $V$ of $x$ such that $x \in V \subseteq U$, and for $y \in V$, there is a slight homeomorphism $f:(X, \tau) \rightarrow(X, \tau)$ such that $f(x)=y$ and $f(t)=t$ for $t \in X-V$. By Proposition 1.4, $f$ is a homeomorphism and the result follows.

THEOREM 2.7. Every zero dimensional SCDH space is SSLH.

Proof. Let $(X, \tau)$ be a zero dimensional SCDH space. Then by Propositions 1.7 and $1.8(\mathrm{~b})$, and Theorem 2.7, it follows that $(X, \tau)$ is SSLH.

THEOREM 2.8. Every zero dimensional slightly homogeneous $T_{0}$ space is SSLH.

Proof. Let $(X, \tau)$ be a zero dimensional slightly homogeneous $T_{0}$ space. Then by Proposition 1.9 it is homogeneous. Thus, by Proposition $1.10(X, \tau)$ is SLH. Hence by Theorem $2.5,(X, \tau)$ is SSLH.

Authors in [19] show that the space $\left(\mathbb{Q}, \tau_{u}\right)$ is not SCDH. On the other hand, it is well known that $\left(\mathbb{Q}, \tau_{u}\right)$ is a zero dimensional $T_{0}$ space and homogeneous. Therefore, by Theorem 2.8 , it is SSLH. So, SSLH spaces are not $\mathrm{SCDH}$ in general.

THEOREM 2.9. Let $(X, \tau)$ be an SSLH space. Then every slightly homogeneous component of $(X, \tau)$ is a clopen subset of $X$.

Proof. Let $x \in X$. Let $y \in S C_{x}$ and let $U$ be an open subset of $X$ with $y \in U$. Then there exists an open set $V$ such that $y \in V \subseteq U$ and for $z \in V$ there is a slight homeomorphism $f:(X, \tau) \rightarrow(X, \tau)$ such that $f(y)=z$ and $f(t)=t$ for all $t \in X-V$. Thus, $y \in V \subseteq S C_{x}$ and so $S C_{x}$ is open. Therefore, slightly homogeneous components are open. On the other hand, since slightly homogeneous components form a partition of $X$, they are clopen subsets of $X$.

THEOREM 2.10. Every clopen subspace of an SSLH space is SSLH.

Proof. Let $(X, \tau)$ be an SSLH space and let $\left(A, \tau_{A}\right)$ be a clopen subspace of $(X, \tau)$. Let $y \in A$ and $U$ be an open subset of $\left(A, \tau_{A}\right)$ such that $y \in U$. Then $U$ is open in $X$ and hence there exists an open set $V \subseteq X$ with $y \in V \subseteq U$, and if $z \in V$, there exists a slight homeomorphism $h:(X, \tau) \rightarrow(X, \tau)$ such that $h(y)=z$ and $h(t)=t$ for all $t \in X-V$. Since $h(A)=A$, we can define $g:\left(A, \tau_{A}\right) \rightarrow\left(A, \tau_{A}\right)$ to be the restriction of $h$ to $A$. Then by Proposition 1.11, $g$ is a slight homeomorphism. Moreover, $g(y)=z$ and $g(t)=t$ for all $t \in$ $A-V$

COROLLARY 2.11. Let $(X, \tau)$ be an SSLH space. If $S C_{x}$ is a slightly homogeneous component of $(\mathrm{X}, \tau)$, then $S C_{x}$ is SSLH.

Proof. Let $S C_{x}$ be a slightly homogeneous component. According to Theorem 2.9, $S C_{x}$ is clopen in X. Hence, by Theorem 2.10, $S C_{x}$ is SSLH.

THEOREM 2.12. Let $\left\{\left(X_{\alpha}, \tau_{\alpha}\right): \alpha \in \Lambda\right\}$ be a family of SSLH spaces such that $X_{\alpha} \cap X_{\beta}=\emptyset$ for all $\alpha \neq \beta$. Then the disjoint sum space $\left(\mathrm{U}\left\{X_{\alpha}: \alpha \in \Lambda\right\}, \tau_{d}\right)$ is SSLH.

Proof. Let $p \in \cup\left\{X_{\alpha}: \alpha \in \Lambda\right\}$ and $U$ be an open subset of $U\left\{X_{\alpha}: \alpha \in \Lambda\right\}$ such that $p \in U$. Then there exists $\beta \in \Lambda$, such that $p \in X_{\beta}$. Let $V=U \cap X_{\beta}$. Then $V$ is open in $\bigcup\left\{X_{\alpha}: \alpha \in \Lambda\right\}$ such that $p \in V$. Since $X_{\beta}$ is SSLH, there exists an open set $W$ such that $p \in W \subseteq V \subseteq U$ such that for every $q \in W$, there exists a slight homeomorphism $h_{\beta}:\left(X_{\beta}, \tau_{\beta}\right) \rightarrow\left(X_{\beta}, \tau_{\beta}\right)$ such that $h_{\beta}(p)=q$ and $h_{\beta}(x)=x$ for $x \in X_{\beta}-W$. Note that $W$ is open in $\bigcup\left\{X_{\alpha}: \alpha \in \Lambda\right\}$ and $p \in W \subseteq U$. Let $q \in W$, then there exists a slight homeomorphism $h_{\beta}:\left(X_{\beta}, \tau_{\beta}\right) \rightarrow\left(X_{\beta}, \tau_{\beta}\right)$ such that $h_{\beta}(p)=q$ and $h_{\beta}(x)=x$ for $x \in X_{\beta}-W$. Define $h:\left(U\left\{X_{\alpha}: \alpha \in\right.\right.$ $\left.\Lambda\}, \tau_{d}\right) \rightarrow\left(\cup\left\{X_{\alpha}: \alpha \in \Lambda\right\}, \tau_{d}\right)$ by $h(x)=h_{\beta}(x)$ if $x \in X_{\beta}$ and $h(x)=x$ if $x \in \cup\left\{X_{\alpha}-X_{\beta}: \alpha \in \Lambda\right\}$. Then by Proposition 1.12, h is a slight homeomorphism. Also $h(p)=q$ and $h(t)=t$ for all $t \in \bigcup\left\{X_{\alpha}-V: \alpha \in \Lambda\right\}$. Thus, $\left(U\left\{X_{\alpha}: \alpha \in \Lambda\right\}, \tau_{d}\right)$ is SSLH.

\section{SLIGHTLY DENSE SETS}

Definition 3.1. Let $(X, \tau)$ be a space. A subset $A \subseteq X$ is said to be slightly dense if for every non-empty clopen set $U \subseteq X, U \cap A \neq \emptyset$.

THEOREM 3.2. Let $(X, \tau)$ be a space. Then every dense subset of $X$ is slightly dense. 
Proof. Let $(X, \tau)$ be a space and let $D$ be a dense subset of $X$. Let $U$ be a clopen subset of $X$. Then $D \cap U \neq$ $\emptyset$. Hence, $D$ is slightly dense.

The converse of Theorem 3.2 is not true in general as the following example shows.

Example 3.3. Consider the space $\left((0,1) \cup(2,3), \tau_{u}\right)$. Let $D=\{(1 / 2),(5 / 2)\}$. Since the only proper clopen sets in $\left((0,1) \cup(2,3), \tau_{u}\right)$ are $(0,1),(2,3)$ and $D \cap(0,1) \neq \emptyset, D \cap(2,3) \neq \emptyset$, it follows that $D$ is a slightly dense set. On the other hand, $D$ is not dense because $D \cap((1 / 4), 1)=\emptyset$.

The following theorem gives us a sufficient condition on slightly dense sets to be dense.

THEOREM 3.4. Let $(X, \tau)$ be a zero dimensional space and let $D \subseteq X$. Then $D$ is dense in $X$ iff it is slightly dense in $X$.

Proof. $\Rightarrow$ ) Theorem 3.2.

$\Leftarrow)$ Suppose that $D$ is a slightly dense subset of $X$. Let $U$ be a non-empty open subset of $X$. Take $x \in U$ and take a clopen basic open set $B$ such that $x \in B \subseteq U$. Since $D$ is slightly dense, $D \cap B \neq \emptyset$. Thus, $D \cap U \neq$ $\emptyset$ and hence $D$ is dense.

THEOREM 3.5. The slightly continuous image of a slightly dense set is slightly dense.

Proof. Let $f:\left(X, \tau_{1}\right) \rightarrow\left(Y, \tau_{2}\right)$ be a slightly continuous function and let $D$ be a slightly dense subset of $\left(X, \tau_{1}\right)$. To show that $f(D)$ is slightly dense, let $V$ be a non-empty clopen subset of $Y$. Then $f^{-1}(V)$ is a nonempty clopen subset of $X$. Since $D$ is slightly dense, $f^{-1}(V) \cap D \neq \emptyset$ and so $V \cap f(D) \neq \emptyset$ and hence $f(D)$ is slightly dense.

THEOREM 3.6. Let $(X, \tau)$ be a space such that for all $x \in X, S C_{x}$ is not open. Then $X-S C_{x}$ is slightly dense in $(X, \tau)$.

Proof. Suppose to the contrary that for some $x \in X, S C_{x}$ is not slightly dense, then there exists a non-empty clopen set $U \subseteq X$ such that $U \cap\left(X-S C_{x}\right)=\emptyset$. Then $U \subseteq S C_{x}$. Therefore, by Proposition 1.13, it follows that $S C_{x}$ is open, a contradiction.

Definition 3.7. For every finite non zero cardinal number $n$, denote the set $\{A \subseteq$ $X: A$ is slightly dense and $|A|=n\}$ by $C_{n}$, and denote the set $\{A \subseteq X: A$ is slightly dense and $|A|=$ $\left.\aleph_{0}\right\}$ by $C_{\infty}$.

THEOREM 3.8. Let $(X, \tau)$ be a space. Then the following are equivalent:

(i) $(X, \tau)$ is connected.

(ii) $A$ is slightly dense for all $A \subseteq X$.

(iii) $\{x\}$ is slightly dense for all $x \in X$.

(iv) $C_{1} \neq \emptyset$.

Proof. (i) $\Rightarrow$ (ii) Let $A$ be a subset of $X$ and let $U$ be a non-empty clopen set in $X$. Then $U=X$ and so $A \cap$ $U \neq \emptyset$. Therefore, $A$ is slightly dense.

(ii) $\Rightarrow$ (iii) Obvious.

(iii) $\Rightarrow$ (iv) Obvious.

(iv) $\Rightarrow$ (i) Suppose to the contrary that there exists a non-empty clopen proper subset $U \subseteq X$. Take $x_{0} \in X$ such that $\left\{x_{0}\right\} \in C_{1}$. Then $\left\{x_{0}\right\} \cap U \neq \emptyset$ and $\left\{x_{0}\right\} \cap(X-U) \neq \varnothing$, a contradiction.

THEOREM 3.9. Let $(X, \tau)$ be a disconnected space. Suppose that for all $n$-tons $A_{n} \subseteq X, A_{n} \in$ $C_{n}$. Then $|X| \leq 2 n-2$.

Proof. Take a non-empty proper clopen subset $U \subseteq X$.

Claim. $|U| \leq n-1$ and $|X-U| \leq n-1$.

Proof of claim. If $|U| \geq n$, take $n$-tons $A_{n} \subseteq U$. By hypothesis, $A_{n}$ is slightly dense and so $A_{n} \cap(X-U) \neq$ $\emptyset$, absurd. Similarly, we can see that $|X-U| \leq n-1$. Therefore, $|X| \leq 2 n-2$.

COROLLARY 3.10. Let $(X, \tau)$ be a disconnected space. Suppose that for all 2-tons in $X, A_{2} \in C_{2}$. Then $|X|=2$ and $\tau=\tau_{\text {disc }}$.

Proof. By Theorem 3.9, $|X| \leq 2$. Since $(X, \tau)$ is disconnected, $|X|=2$ and $\tau=\tau_{\text {disc }}$.

COROLLARY 3.11. If $X$ is a set with $|X|>2$ and $\tau$ is a topology on $X$, then $(X, \tau)$ is connected iff $\{x, y\} \in$ $C_{2}$ for all $x, y \in X$ with $x \neq y$.

Proof. $\Rightarrow$ ) Theorem 3.8.

$\Leftarrow)$ Corollary 3.10 .

The following example shows that if $C_{2} \neq \varnothing$, then it is not necessarily true that $\{x, y\} \in C_{2}$ for all $x, y \in X$ with $x \neq y$.

Example 3.12. Let $X=\{a, b, c\}$ and $\tau=\{\varnothing, X,\{a\},\{b, c\}\}$. Then $\{a, b\}$ is slightly dense but $\{b, c\}$ is not.

COROLLARY 3.13. Let $(X, \tau)$ be a space such that for all $n$-tons $A_{n} \subseteq X, A_{n} \in C_{n}$, and $|X|>2 n-2$. Then $(X, \tau)$ is connected.

Definition 3.14. A space $(X, \tau)$ is said to be slightly separable if it contains a countable slightly dense subset.

PROPOSITION 3.15. A space $(X, \tau)$ is slightly separable iff $C_{n} \neq \emptyset$ for some $n \in \mathbb{N} \cup\{\infty\}$.

PROPOSITION 3.16. Every connected space is slightly separable. 
Proof. Let $(X, \tau)$ be a connected space. Then by Theorem 3.8, $C_{1} \neq \emptyset$ and so by Proposition 3.15, it follows that $(X, \tau)$ is slightly separable.

The discrete space on any countable set $X$ with $|X|>1$ is a slightly separable disconnected space. This shows that the converse of Proposition 3.16 is not true in general.

PROPOSITION 3.17. Every separable space is slightly separable.

Proof. Let $(X, \tau)$ be a separable space. Choose a countable dense subset $D \subseteq X$. Then by Theorem 3.2 it follows that $D$ is slightly dense. Therefore, $(X, \tau)$ is slightly separable space.

The space $\left(\mathbb{R}, \tau_{c o c}\right)$ where $\tau_{\text {coc }}$ is the cocountable topology on $\mathbb{R}$, shows that the converse of Proposition 3.17 is not true in general.

PROPOSITION 3.18. Let $(X, \tau)$ be a zero dimensional space. Then $(X, \tau)$ is separable iff $(X, \tau)$ slightly separable space.

Proof. $\Rightarrow$ ) Proposition 3.17.

$\Leftarrow)$ Let $D$ be a countable slightly dense subset of $X$. Then by Theorem 3.4, $D$ is dense.

THEOREM 3.19. The slightly continuous image of a slightly separable space is slightly separable.

Proof. Let $f:\left(X, \tau_{1}\right) \rightarrow\left(Y, \tau_{2}\right)$ be a slightly continuous function with $\left(X, \tau_{1}\right)$ is slightly separable. Let $D$ be a countable slightly dense subset of $X$. Then by Theorem $3.5 f(D)$ is slightly dense. Furthermore, $f(D)$ is countable and hence $\left(Y, \tau_{2}\right)$ is slightly separable.

COROLLAY 3.20. Being "slightly separable" is a slightly topological property.

COROLLARY 3.21. Being "slightly separable" is a topological property.

THEOREM 3.22. A clopen subspace of a slightly separable space is slightly separable.

Proof. Let $(X, \tau)$ be a slightly separable space. Let $A$ be a clopen subset of $X$. Let $S$ be a countable slightly dense subset of $(X, \tau)$. Then $S \cap A$ is a slightly dense subset of $\left(A, \tau_{A}\right)$. Indeed, let $U$ be a non-empty clopen subset of $\left(A, \tau_{A}\right)$. Since $U$ is clopen in the clopen subspace $\left(A, \tau_{A}\right)$, $\mathrm{U}$ is clopen in $(\mathrm{X}, \tau)$. Therefore, $U \cap A$ is clopen in $(\mathrm{X}, \tau)$ and $S \cap(A \cap U) \neq \emptyset$. Hence $(S \cap A) \cap U \neq \emptyset$. Therefore, $S \cap A$ is a slightly dense subset of $\left(A, \tau_{A}\right)$.

THEOREM 3.23. Let $\left\{\left(X_{\alpha}, \tau_{\alpha}\right): \alpha \in \Lambda\right\}$ be a family of spaces such that $X_{\alpha} \cap X_{\beta}=\emptyset$ for all $\alpha \neq \beta$. If for all $\alpha \in \Lambda,\left(X_{\alpha}, \tau_{\alpha}\right)$ contains a non-empty slightly dense set $D_{\alpha}$, then $U\left\{D_{\alpha}: \alpha \in \Lambda\right\}$ is slightly dense in the disjoint sum space $\left(\bigcup\left\{X_{\alpha}: \alpha \in \Lambda\right\}, \tau_{d}\right)$.

Proof. Let $U$ be a non-empty clopen set in the disjoint sum space $\left(\bigcup\left\{X_{\alpha}: \alpha \in \Lambda\right\}, \tau_{d}\right)$. Then there exists $\beta \in$ $\Lambda$ such that $U \cap X_{\beta} \neq \varnothing$. Since $D_{\beta}$ is slightly dense in $X_{\beta}, D_{\beta} \cap\left(U \cap X_{\beta}\right) \neq \emptyset$. Thus, $\left(\cup\left\{D_{\alpha}: \alpha \in \Lambda\right\}\right)$ $\cap U \neq \varnothing$.

COROLLARY 3.24. Let $\left\{\left(X_{n}, \tau_{n}\right): n \in \mathbb{N}\right\}$ be a countable family of spaces with $X_{n} \cap X_{m}=\emptyset$ for $n \neq m$. If for all $n \in \mathbb{N},\left(X_{n}, \tau_{n}\right)$ is slightly separable spaces, then the disjoint sum space $\left(\cup\left\{X_{n}: n \in \mathbb{N}\right\}, \tau_{d}\right)$ is slightly separable.

Proof. For every $n \in \mathbb{N}$, choose a countable slightly dense set $D_{n} \subseteq X_{n}$. Then $\bigcup\left\{D_{n}: n \in \mathbb{N}\right\}$ is countable. Moreover, By Theorem 3.23, it follows that $\bigcup\left\{D_{n}: n \in \mathbb{N}\right\}$ is slightly dense. Thus, $\left(\bigcup\left\{X_{n}: n \in \mathbb{N}\right\}, \tau_{d}\right)$ is a slightly separable space.

THEOREM 3.25. Let $\left\{\left(X_{\alpha}, \tau_{\alpha}\right): \alpha \in \Lambda\right\}$ be a family of spaces and let $\left(\Pi\left\{X_{\alpha}: \alpha \in \Lambda\right\}, \tau_{\text {prod }}\right)$ be the product space. If $\left(\Pi\left\{X_{\alpha}: \alpha \in \Lambda\right\}, \tau_{\text {prod }}\right)$ is slightly separable, then for all $\alpha \in \Lambda,\left(X_{\alpha}, \tau_{\alpha}\right)$ is slightly separable.

Proof. Let $\beta \in \Lambda$. Since the projection $P_{\beta}:\left(\Pi\left\{X_{\alpha}: \alpha \in \Lambda\right\}, \tau_{\text {prod }}\right) \rightarrow\left(\Pi\left\{X_{\alpha}: \alpha \in \Lambda\right\}, \tau_{\text {prod }}\right)$ is continuous, it is slightly continuous. Therefore, by Theorem 3.19 , it follows that $\left(X_{\beta}, \tau_{\beta}\right)$ is slightly separable.

As defined, a space $(X, \tau)$ is called extremally disconnected if the closure of every open set is open. THEOREM 3.26. Let $\left(X, \tau_{1}\right)$ and $\left(Y, \tau_{2}\right)$ be two extremally disconnected spaces. Then the product space $\left(X \times Y, \tau_{\text {prod }}\right)$ is slightly separable iff both $\left(X, \tau_{1}\right)$ and $\left(Y, \tau_{2}\right)$ are slightly separable.

Proof. $\Rightarrow$ ) Theorem 3.25 .

$\Leftarrow)$ Let $D_{X}$ and $D_{Y}$ be countable slightly dense sets in $X$ and $Y$, respectively. Then $D_{X} \times D_{Y}$ is countable. Let $U$ be a non-empty clopen subset of $X \times Y$. Take $(x, y) \in U$ and take $A \in \tau_{1}, B \in \tau_{2}$ such that $(x, y) \in A \times$ $B \subseteq U$. Thus, $A \times B \subseteq U$ and hence $A \times B \subseteq U$. Since $\left(X, \tau_{1}\right)$ and $\left(Y, \tau_{2}\right)$ are extremally disconnected spaces, $A$ and $B$ are clopen. Therefore, $A \cap D_{X} \neq \emptyset$ and $B \cap D_{Y} \neq \emptyset$ and so $\left(D_{X} \times D_{Y}\right) \cap U \neq \emptyset$.

\section{TWO TYPES OF SLIGHTLY COUNTABLE DENSE HOMOGENEOUS SPACES}

Definition 4.1. A space $(X, \tau)$ is said to be slightly countable dense homogeneous of type (2) $(\operatorname{SCDH}(2))$ if

(i) $(X, \tau)$ is slightly separable.

(ii) For any two countable slightly dense sets $A$ and $B$ in $X$, there exists a slight homeomorphism $h:(X, \tau) \rightarrow$ $(X, \tau)$ such that $h(A)=B$.

THEOREM 4.2. If $(X, \tau)$ is $\operatorname{SCDH}(2)$, then every slightly dense subset of $X$ different from $X$ is infinite. 
Proof. Suppose to the contrary that there exists a finite slightly dense set $A \subset X$. Take $x_{0} \in X-A$, let $B=$ $A \cup\left\{x_{0}\right\}$. Then $\mathrm{A}$ and $\mathrm{B}$ are two countable slightly dense sets in $(X, \tau)$, and so there exists a slight homeomorphism $h:(X, \tau) \rightarrow(X, \tau)$ such that $h(A)=B$, a contradiction.

COROLLARY 4.3. Let $(X, \tau)$ be a connected space. Then $(X, \tau)$ is $\operatorname{SCDH}(2)$ iff $|X|=1$.

Proof. $\Rightarrow)$ Suppose to the contrary that $|X|>1$. Take $x_{0} \in X$. Then $\left\{x_{0}\right\}$ is a slightly dense subset of $X$ different from $X$. This contradicts Theorem 4.2.

$\Leftarrow)$ Trivial.

In spite of that every dense set is slightly dense, every separable space is slightly separable and every homeomorphism is slight homeomorphism. A CDH space need not to be $\operatorname{SCDH}(2)$ in general as we will see in this example.

Example 4.4. Consider the space $\left(\mathbb{R}, \tau_{u}\right)$. By Proposition $1.14,\left(\mathbb{R}, \tau_{u}\right)$ is $\mathrm{CDH}$. However, Corollary 4.3 shows that $\left(\mathbb{R}, \tau_{u}\right)$ is not $\operatorname{SCDH}(2)$.

THEOREM 4.5. A zero dimensional space is CDH iff it is $\operatorname{SCDH}(2)$.

Proof. Let $(X, \tau)$ be a CDH zero dimensional space. Then by Proposition $3.18,(X, \tau)$ is slightly separable. Let $\mathrm{A}$ and $B$ be two countable slightly dense subsets of $X$. Then by Theorem 3.4 they are countable dense sets of the $\mathrm{CDH}$ space $(X, \tau)$ and so there exists a homeomorphism $f:(X, \tau) \rightarrow(X, \tau)$ such that $f(A)=B$. By Proposition 1.2 it follows that $\mathrm{f}$ is a slight homeomorphism, and then $(X, \tau)$ is $\operatorname{SCDH}(2)$. Conversely, suppose that $(X, \tau)$ is a zero dimensional $\operatorname{SCDH}(2)$. Then by Proposition 3.18, $(X, \tau)$ is separable. Let $A$ and $B$ be two countable dense subsets of $(X, \tau)$. Then by Theorem $3.2, A$ and $B$ are countable slightly dense subsets of $(X, \tau)$. Since $(X, \tau)$ is $\operatorname{SCDH}(2)$, there exists a slight homeomorphism $h:(X, \tau) \rightarrow(X, \tau)$ such that $h(A)=B$. By Proposition 1.4, it follows that $\mathrm{h}$ is a homeomorphism. Hence $(X, \tau)$ is $\mathrm{CDH}$.

Example 4.6. Consider the space $\left(\mathbb{Q}^{c}, \tau_{u}\right)$. By Proposition $1.15,\left(\mathbb{Q}^{c}, \tau_{u}\right)$ is $\mathrm{CDH}$, and since it is zero dimensional, by the Theorem 4.5 it is $\operatorname{SCDH}(2)$.

LEMMA 4.7. Let $(X, \tau)$ be a space. If $X$ is the only slightly dense set in $X$, then $\tau=\tau_{\text {disc }}$.

Proof. Let $x \in X$. Since $X$ is the only slightly dense set, $X-\{x\}$ is not slightly dense. Thus, there exists a non-empty clopen set $U \subseteq X$ such that $U \cap(X-\{x\})=\emptyset$. Therefore, $U=\{x\}$ and hence $\tau=\tau_{\text {disc }}$.

THEOREM 4.8. Let $(X, \tau)$ be a $\operatorname{SCDH}(2)$ space. Then $X$ is countable iff $\tau=\tau_{\text {disc }}$.

Proof. $\Rightarrow)$ Let $A$ be a slightly dense subset of $X$. Then there exists a slight homeomorphism $f:(X, \tau) \rightarrow(X, \tau)$ such that $f(A)=X=f(X)$ and so $A=X$. Thus, the only slightly dense subset of $(X, \tau)$ is $X$, and hence by Lemma 4.7, $\tau=\tau_{\text {disc }}$.

$\Leftarrow)$ Suppose to the contrary that $X$ is uncountable. Take a countable slightly dense set $A \subseteq X$. Then $A=X$, a contradiction.

Example 4.9. The space $\left(\mathbb{Q}, \tau_{u}\right)$ is not a discrete space. Since $\mathbb{Q}$ is countable, by Theorem 4.8 , it follows that $\left(\mathbb{Q}, \tau_{u}\right)$ is not $\operatorname{SCDH}(2)$.

THEOREM 4.10. Let $(X, \tau)$ be a zero dimensional space. Then $(X, \tau)$ is $\operatorname{SCDH}(2)$ iff it is $\operatorname{SCDH}$.

Proof. $\Rightarrow)$ By Theorem $4.5,(X, \tau)$ is CDH. Thus by Proposition $1.8(\mathrm{a}),(X, \tau) \mathrm{SCDH}$.

$\Leftarrow)$ By Proposition $4.5(\mathrm{~b}),(X, \tau)$ is $\mathrm{CDH}$. Thus by Theorem $4.5(X, \tau)$ is $\operatorname{SCDH}(2)$.

The space $\left(\mathbb{R}, \tau_{u}\right)$ is $\mathrm{CDH}$ and hence $\operatorname{SCDH}$, also, we show that $\left(\mathbb{R}, \tau_{u}\right)$ is not $\operatorname{SCDH}(2)$. So, in Theorem 4.2.10, the condition "zero dimensional" cannot be dropped.

COROLLARY 4.11. For a zero dimensional space $(X, \tau)$, the following are equivalent:

a) $(X, \tau)$ is $\mathrm{CDH}$.

b) $(X, \tau)$ is SCDH.

c) $(X, \tau)$ is $\operatorname{SCDH}(2)$.

THEOREM 4.12. Being " $\mathrm{SCDH}(2)$ " is a slightly topological property.

Proof. Let $\left(X, \tau_{1}\right)$ be a $\operatorname{SCDH}(2)$ and let $\left(Y, \tau_{2}\right)$ be any space slightly homeomorphic to $\left(X, \tau_{1}\right)$. Let $f:\left(X, \tau_{1}\right) \rightarrow\left(Y, \tau_{2}\right)$ be a slight homeomorphism. Then by Theorem 3.19, $\left(Y, \tau_{2}\right)$ is slightly separable. Let $S_{1}$ and $S_{2}$ be two countable slightly dense sets in $Y$. Then by Theorem $3.5, f^{-1}\left(S_{1}\right)$ and $f^{-1}\left(S_{2}\right)$ are countable slightly dense sets in $X$. Since $\left(X, \tau_{1}\right)$ is $\operatorname{SCDH}(2)$, there exists a slight homeomorphism $h:\left(X, \tau_{1}\right) \rightarrow\left(X, \tau_{1}\right)$ such that $h\left(f^{-1}\left(S_{1}\right)\right)=f^{-1}\left(S_{2}\right)$. Define $g:\left(Y, \tau_{2}\right) \rightarrow\left(Y, \tau_{2}\right)$ by $g(y)=\left(f \circ h \circ f^{-1}\right)(y)$, then $g$ is a slight homeomorphism takes $S_{1}$ to $S_{2}$. Therefore, $\left(Y, \tau_{2}\right)$ is $\operatorname{SCDH}(2)$.

COROLLAY 4.13. Being "SCDH(2)" is a topological property.

THEOREM 4.14. Let $\left\{\left(X_{n}, \tau_{n}\right): n \in \mathbb{N}\right\}$ be a family of $\operatorname{SCDH}(2)$ spaces with $X_{n} \cap X_{m}=\emptyset$ for $n \neq m$. Then the disjoint sum space $\left(\bigcup\left\{X_{n}: n \in \mathbb{N}\right\}, \tau_{d}\right)$ is $\operatorname{SCDH}(2)$.

Proof. Since for each $n \in \mathbb{N},\left(X_{n}, \tau_{n}\right)$ is slightly separable, by Corollary 3.24 , (U $\left.\left\{X_{n}: n \in \mathbb{N}\right\}, \tau_{d}\right)$ is slightly separable. Let $S_{1}$ and $S_{2}$ be two countable slightly dense sets in $\bigcup\left\{X_{n}: n \in \mathbb{N}\right\}$, then for $n=1,2, \ldots S_{1} \cap X_{n}$ and $S^{2} \cap X_{n}$ are countable slightly dense sets in $X_{n}$ and so there is a slight homeomorphism $f_{n}:\left(X_{n}, \tau_{n}\right) \rightarrow$ $\left(X_{n}, \tau_{n}\right)$ such that $f_{n}\left(S_{1} \cap X_{n}\right)=S_{2} \cap X_{n}$. Define $h:\left(\bigcup\left\{X_{n}: n \in \mathbb{N}\right\}, \tau_{d}\right) \rightarrow\left(\bigcup\left\{X_{n}: n \in \mathbb{N}\right\}, \tau_{d}\right)$ such that if 
$x \in X_{n}$, then $h(x)=f_{n}(x)$. Then $h$ is a slight homeomorphism such that $h\left(S_{1}\right)=S_{2}$. It follows that $\left(\mathrm{U}\left\{X_{n}: n \in \mathbb{N}\right\}, \tau_{d}\right)$ is $\operatorname{SCDH}(2)$.

Definition 4.15. A space $(X, \tau)$ is said to be slightly countable dense homogeneous of type (3) (SCDH(3)) if

(i) $(X, \tau)$ is slightly separable.

(ii) If $n \in \mathbb{N} \cup\{\infty\}$ and $A, B \in C_{n}$, then there exists a slight homeomorphism $f:(X, \tau) \rightarrow(X, \tau)$ such that $f(A)=B$.

THEOREM 4.16. Every connected space is $\operatorname{SCDH}(3)$.

Proof. Let $(X, \tau)$ be a connected space. Then by Theorem $3.16,(X, \tau)$ is slightly separable. Let $n \in \mathbb{N} \cup\{\infty\}$ and let $A, B \in C_{n}$. Choose a bijection $f:(X, \tau) \rightarrow(X, \tau)$ with $f(A)=B$. Since $(X, \tau)$ is connected, $f$ is a slight homeomorphism and hence $(X, \tau)$ is $\operatorname{SCDH}(3)$.

THEOREM 4.17. Every $\operatorname{SCDH}(2)$ space is $\operatorname{SCDH}(3)$.

Proof. Let $(X, \tau)$ be a $\operatorname{SCDH}(2)$. Then $(X, \tau)$ is slightly separable. Let $n \in \mathbb{N} \cup\{\infty\}$ and let $A, B \in C_{n}$. Then $A, B$ are countable slightly dense subsets of $(X, \tau)$, and hence there is a slight homeomorphism $f:(X, \tau) \rightarrow$ $(X, \tau)$ such that $f(A)=B$.

The converse of Theorem 4.17 is not true in general. For example, $\left(\mathbb{R}, \tau_{u}\right)$ is $\operatorname{SCDH}(3)$ but not $\operatorname{SCDH}(2)$

THEOREM 4.18. Every zero dimensional CDH space is $\mathrm{SCDH}(3)$.

Proof. Let $(X, \tau)$ be a zero dimensional $\mathrm{CDH}$ space. Then by Theorem $4.5(X, \tau)$ is $\operatorname{SCDH}(2)$ and by Theorem 4.17 it is $\operatorname{SCDH}(3)$.

Since the space $\left(\mathbb{Q}^{c}, \tau_{u}\right)$ is zero dimensional and CDH space, by Theorem 4.18 it is $\operatorname{SCDH}(3)$.

THEOREM 4.19. Let $(X, \tau)$ be a space such that all slightly dense sets in $X$ have the same cardinality, then $(X, \tau)$ is $\operatorname{SCDH}(2)$ iff it is $\operatorname{SCDH}(3)$.

Proof. $\Rightarrow$ ) Theorem 4.17 .

$\Leftarrow)$ Let $A$ and $B$ be two countable slightly dense sets in $X$. Then $|A|=|B|$ and since $(X, \tau)$ is $\operatorname{SCDH}(3)$, we get the result.

Example 4.20. Let $X=\{a, b, c\}$ and $\tau=\{\varnothing, X,\{a\},\{b, c\}\}$. Then $(X, \tau)$ is a $\operatorname{SCDH}(3)$ space. Also, Since $X$ is countable and $\tau \neq \tau_{\text {disc }}$, by Theorem 4.8 it is not $\operatorname{SCDH}(2)$. Since $(X, \tau)$ is zero dimensional, by Theorem 4.5 it is not $\mathrm{CDH}$.

THEOREM 4.21. Being "SCDH(3)" is a slightly topological property.

Proof. Let $\left(X, \tau_{1}\right)$ be a $\operatorname{SCDH}(3)$ and let $\left(Y, \tau_{2}\right)$ be any space slightly homeomorphic to $\left(X, \tau_{1}\right)$. Let $f:\left(X, \tau_{1}\right) \rightarrow\left(Y, \tau_{2}\right)$ be a slight homeomorphism. Then by Corollary 3.20, $\left(Y, \tau_{2}\right)$ is slightly separable. Let $S_{1}$ and $S_{2}$ be two countable slightly dense sets in $Y$ such that $\left|S_{1}\right|=\left|S_{2}\right|$. Then by Theorem $3.5 f^{-1}\left(S_{1}\right)$ and $f^{-1}\left(S_{2}\right)$ are countable slightly dense sets in $X$ with $\left|f^{-1}\left(S_{1}\right)\right|=\left|f^{-1}\left(S_{2}\right)\right|$. Since $\left(X, \tau_{1}\right)$ is $\operatorname{SCDH}(3)$, there exists a slight homeomorphism $h:\left(X, \tau_{1}\right) \rightarrow\left(X, \tau_{1}\right)$ such that $h\left(f^{-1}\left(S_{1}\right)\right)=f^{-1}\left(S_{2}\right)$. Define $g:\left(Y, \tau_{2}\right) \rightarrow$ $\left(Y, \tau_{2}\right)$ by $g(y)=\left(f \circ h \circ f^{-1}\right)(y)$. Then $\mathrm{g}$ is a slight homeomorphism takes $S_{1}$ to $S_{2}$. Thus, $\left(Y, \tau_{2}\right)$ is $\operatorname{SCDH}(3)$.

COROLLAY 4.22. Being "SCDH(3)" is a topological property.

\section{REFERENCES}

[1] W. Sierpenski, "Sur uni proprit'te' topologique des ensembles de'nombrable dense en soi," Fundamenta Mathematicae, vol. 1, pp. 11-28, 1920.

[2] L. Ford Jr., "Homeomorphism groups and coset spaces," Transactions of the American Mathematical Society, vol. 77, pp. 490-497, 1954.

[3] R. Bennett, "Countable dense homogeneous spaces," Fundamenta Mathematicae, vol. 74, pp. 189-194, 1972.

[4] S. Al Ghour, "SLH fuzzy spaces," African Diaspora Journal of Mathematics, vol. 2, pp. 61-67, 2004.

[5] S. Al Ghour and A. Fora, "Minimality and homogeneity in fuzzy spaces," Journal of Fuzzy Mathematics, vol 12, pp. 725-737, 2004.

[6] S. Al Ghour, A. Al-Omari, and T. Noiri, "On homogeneity and homogeneity components in generalized topological spaces," Filomat, vol. 27, no. 6, pp. 1097-1105, 2013.

[7] M. Hrušák and J. van Mill, "Nearly countable dense homogeneous spaces," Canadian Journal of Mathematics, vol. 66, no. 4, pp. 743-758, 2014.

[8] J. van Mill, "Countable dense homogeneous rimcompact spaces and local connectivity," Filomat, vol. 29, no. 1, pp. 179-182, 2015.

[9] S. Al Ghour and A. Fora, "On CDH fuzzy spaces," Journal of Intelligent and Fuzzy Systems, vol. 30, no. 2, pp. 935-941, 2016.

[10] S. Al Ghour and A. Azaizeh, "Fuzzy homogeneous bitopological Spaces," International Journal of Electrical and Computer Engineering (IJECE), vol. 8, no. 1, pp. 2088-8708, 2018.

[11] S. Al Ghour and R. Al-Deiakeh, " $n$-homogeneous and $L H$ generalized topological spaces," Iranian Journal of Science and Technology, Transactions A: Science, vol. 42, pp. 73-79, 2018. 
[12] S. Al Ghour, "Homogeneous components of a CDH fuzzy space," International Journal of Electrical and Computer Engineering (IJECE), vol. 9, no. 4, pp. 2691-2694, 2019.

[13] S. Al Ghour, "Densely homogeneous fuzzy spaces," International Journal of Electrical and Computer Engineering (IJECE), vol. 9, no. 4, pp. 3256-3261, 2019.

[14] S. Al Ghour and A. Azaizeh, "Fuzzy $n$-s-homogeneity and fuzzy weak $n$-s-homogeneity," International Journal of Electrical and Computer Engineering (IJECE), vol. 9, no. 6, pp. 5395-5399, 2019.

[15] S. Al Ghour and A. Bin-Saadon, "On some generated soft topological spaces and soft homogeneity," Heliyon, vol. 5, no.7, pp. 1-10, 2019.

[16] P. Sini, "Hereditarily homogeneous generalized topological spaces," Iranian Journal of Mathematical Sciences and Informatics, vol. 14, no. 2, pp. 9-18, 2019.

[17] S. Lazaar and H. Sabri, "The topological group of autohomeomorphisms of homogeneous functionally Alexandroff spaces," Topology Proceedings, vol. 55, pp. 243-264, 2020.

[18] Singal and R. Jain, "Slightly continuous mappings," Journal of the Indian Mathematical Society (N.S.), vol. 64, pp. 195-203, 1997.

[19] S. Al Ghour and N. Al Khatib, "On slight homogeneous and countable dense homogeneous spaces," Matematički Vesnik, vol. 63, no. 2, pp. 133-144, 2011.

[20] S. Al Ghour, "Minimality and prehomogeneity," Acta Mathematica Universitatis Comenianae (N.S.), vol. 72, pp. 237-244, 2003.

[21] S. Al Ghour, "Components and local prehomogeneity," Acta Mathematica Universitatis Comenianae (N.S.), vol. 73, pp. 187-196, 2004.

[22] S. Al Ghour, K. Zoubi and A. Fora, "Semihomogeneous topological spaces," Publicationes Mathematicae Debrecen, vol. 67, pp. 131-143, 2005.

[23] M. Yassien, "Homogeneous spaces," Master's Thesis, Department of Mathematics, Yarmouk University, Irbid-Jordan, 1995.

[24] Jr. Fitzpatrick and H. Zhou, "A survey of some homogeneity properties in topology," Annals of the New York Academy of Sciences, vol. 552, no. 1, pp. 28-35, 1989.

[25] R. Engelking, "General topology," Hilderman-Berlin, 1989. 